\title{
One-, Two-, and Multi-Fold Origami Axioms
}

\author{
Roger C. Alperin and Robert J. Lang
}

December 6, 2006

\section{Introduction}

In 1989 [15], Huzita introduced the six origami operations that have now become know as the Huzita Axioms (HAs). The HAs, shown in Figure 1, constitute six distinct ways of defining a single fold by bringing together combinations of preexisting points (e.g., crease intersections) and preexisting lines (creases and/or the fold line itself).

It has been shown that all of the standard compass-and-straightedge constructions of Euclidean geometry can be constructed using the original 6 axioms. In fact, working independently, Martin [25] showed that the operation equivalent to Huzita's O6 (plus the definition of a point as a crease intersection) was, by itself, sufficient for the construction of all figures constructible by the full 6 axioms and that this included all compass-and-straightedge constructions. Conversely, Auckly and Cleveland [5, 14], unaware of O5, O6, showed that without O5, O6, the field of numbers constructible by the other 4 HAs was smaller than the field of numbers constructible by compass and straightedge. An analysis of the hierarchy of fields which can be constructed using different axioms systems is detailed in [2], [4].

We note that since the other 5 of the 6 HAs can be constructed using only O6, the derived operations should perhaps be called something other than axioms. However, we will bow to 20 years of established usage and continue to call them axioms.

In the same proceedings that Huzita's original listing appeared, Justin [21] presented a list of seven distinct operations - which Justin credited, in part, to Peter Messer - including one that had been overlooked by Huzita. (A shorter list of 5 operations was also presented by Huzita and Scimemi [19].) Justin's longer listing has been somewhat overlooked, but in 2001, Hatori [13] 


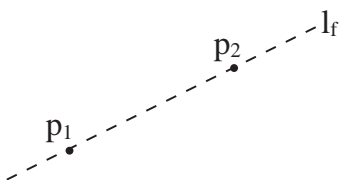

(O1) Given two points $p_{1}$ and $p_{2}$, we can fold a line connecting them.

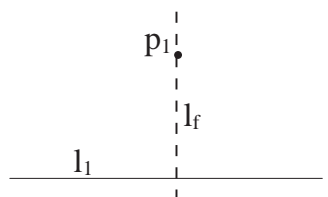

(O4) Given a point $p_{1}$ and a line $l_{1}$, we can make a fold perpendicular to $l_{1}$ passing through the point $p_{1}$.

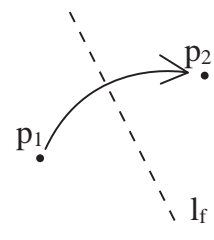

(O2) Given two points $p_{1}$ and $p_{2}$, we can fold $p_{1}$ onto $p_{2}$.

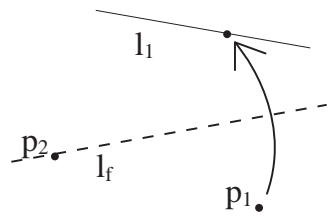

(O5) Given two points $p_{1}$ and $p_{2}$ and a line $l_{1}$, we can make a fold that places $p_{1}$ onto $l_{1}$ and passes through the point $p_{2}$.

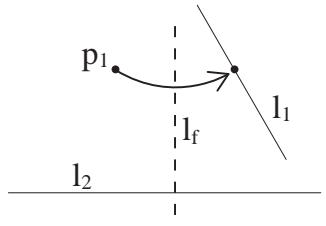

(O7) Given a point $p_{1}$ and two lines $l_{1}$ and $l_{2}$, we can make a fold perpendicular to $l_{2}$ that places $p_{1}$ onto line $l_{1}$.

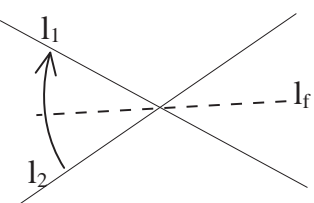

(O3) Given two lines $l_{1}$ and $l_{2}$, we can fold line $l_{1}$ onto $l_{2}$.

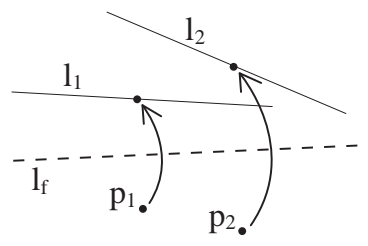

(O6) Given two points $p_{1}$ and $p_{2}$ and two lines $l_{1}$ and $l_{2}$, we can make a fold that places $p_{1}$ onto line $l_{1}$ and places $p_{2}$ onto line $l_{2}$.

Figure 1: O1-O6 are the six "Huzita" axioms. O7 is Justin's (Hatori's) seventh axiom. 
rediscovered Justin's 7th operation, also shown in Figure 1. While similar to the $6 \mathrm{HAs}$, it was was not equivalent to any one of them. However, it did not expand the field of origami-constructible numbers beyond the field of the original 6 HAs. The set of all 7 operations might be called the HuzitaJustin Axioms (HJAs). Hatori's rediscovery raised an interesting question implied by Justin's original list: are the 7 axioms complete, or are there other undiscovered single-fold axioms to be found?

Over the years, various workers have shown many elegant constructions possible with the HJAs, including constructions not possible with compassand-straightedge such as angle trisection [1,7,22], cube doubling [26], and various regular polygons $[9,10,11,12]$. However, there remain constructions that are not possible with the HJAs, such as angle quintisection, the regular 11-gon (the smallest regular polygon not possible with the HJAs), or solution of the general quintic equation.

One of us [23] recently demonstrated an angle quintisection obtained by folding alone. However, this construction lies outside of the field of HJAconstructions in that at one step it requires making two simultaneous creases, while all of the HJAs involve making only a single crease. As a consequence of well-known results from field theory [27, p.170], [8, p. 450], this leads to a construction of a regular polygon with 11 sides. More generally, any $n$-sided regular polygon with value $\phi(n)$ of Euler's totient function divisible by only 2,3 or 5 can be constructed using folding operations involving the HJA constructions or the multiple crease quintisection.

This raises the question: if we consider making 2, 3, or more simultaneous creases, what types of construction are possible? The angle quintisection demonstrates that at least one irreducible quintic equation can be solved by two-fold operations; what higher orders are possible?

In this work, we investigate both one- and two-fold operations. We first show that all of the HJAs can be described as a combination of one or two more fundamental conditions, which we call alignments. We identify all possible alignments, and then, by exhaustive enumeration, show that the 7 HJAs include all possible combinations of such alignments. In a previous work, one of us [24] noted that the completeness of the HJAs could be shown and private copies of the proof have been circulated. However, this is, to our knowledge, the first public complete proof.

We then turn our attention to two-fold alignments and axioms. We identify a unique set of 17 alignments that may be combined to define two simultaneous creases. We then show how an exhaustive (computer-aided) 
enumeration of all possible alignment combinations leads to 489 distinct operations analogous to the 7 HJAs, but that define two simultaneous creases. We show that the previously-demonstrated angle quintisection utilizes one of these two-fold "axioms". We close by showing that 3 simultaneous folds leads to a solution of the general quintic equation and that, in general, $n-2$ simultaneous folds are sufficient to solve the general $n$ th-degree equation.

\section{Origami Axioms}

In Huzita's original work [15], the HAs were given as literal axioms. In order to prove completeness, we must describe them in terms of more fundamental concepts.

Definition 1 (Point) $A$ point $(x, y)$ is the ordered pair where $x$ and $y$ are the Cartesian coordinates of the point.

Observe that a point has two degrees of freedom (DOF), i.e., it is defined by two numbers - namely, its coordinates.

A line can be described in many ways: slope and intercept, angle and distance from the origin, etc. The following representation offers algebraic simplicity.

Definition 2 (Line) $A$ line $(X, Y)$ is the set of points $(x, y)$ that satisfy the equation $X x+Y y+1=0$.

This definition has the desirable property that every describable line has a unique representation. However, lines passing through the origin cannot be so described. We can deal with this problem in practice by translating any system of points and lines so that no line passes through the origin.

Since both points and lines are represented by ordered pairs, we will adopt the convention that point coordinates are identified with lower-case letters and line coordinates with upper-case letters.

We can now define the folded image of a point or line to be the reflection of the point or line through the fold line. A little algebra gives the following.

Definition 3 (Folded Point) The folded image $F_{L_{F}}(P)$ of a point $P=$ $(x, y)$ in a fold line $L_{F}=\left(X_{F}, Y_{F}\right)$ is the reflection of the point in the fold line. 
In our representation, the folded image of a point is given by

$$
F_{L_{F}}(P)=\left(\frac{x\left(Y_{F}^{2}-X_{F}^{2}\right)-2 X_{F}\left(1+y Y_{F}\right)}{X_{F}^{2}+Y_{F}^{2}}, \frac{y\left(X_{F}^{2}-Y_{F}^{2}\right)-2 Y_{F}\left(1+x X_{F}\right)}{X_{F}^{2}+Y_{F}^{2}}\right) \text {. }
$$

Definition 4 (Folded Line) The folded image $F_{L_{F}}(L)$ of a line $L=(X, Y)$ in a fold line $L_{F}=\left(X_{F}, Y_{F}\right)$ is the reflection of the line in the fold line.

In our representation, the folded image of a line is given by

$$
F_{L_{F}}(L)=\left(\frac{x\left(X_{F}^{2}-Y_{F}^{2}\right)+2 X_{F} Y Y_{F}}{X_{F}^{2}-2 X X_{F}-2 Y Y_{F}+Y_{F}^{2}}, \frac{y\left(X_{F}^{2}-Y_{F}^{2}\right)-2 X X_{F} Y_{F}}{X_{F}^{2}-2 X X_{F}-2 Y Y_{F}+Y_{F}^{2}}\right)
$$

Note that for both points and lines, folding, being the reflection operator, is its own inverse. For any point $P=(x, y)$, line $L=(X, Y)$, and fold line $L_{F}=\left(X_{F}, Y_{F}\right)$, it is easily verified that $F_{L_{F}}\left(F_{L_{F}}(P)\right)=P$ and $F_{L_{F}}\left(F_{L_{F}}(L)\right)=L$. Thus, for any pair of points or lines $A$ and $B$, $F_{L_{F}}(A)=B \Longleftrightarrow F_{L_{F}}(B)=A$.

Each of the HJAs specifies one or more incidences between points, lines, and the folded images of points and/or lines. We call such an incidence an alignment. We denote (co)incidence between two objects $A$ and $B$ by the notation $A \leftrightarrow B$. There are three possible types of alignment:

Definition 5 (Point-Point Alignment) Given two points $P_{1}=\left(x_{1}, y_{1}\right)$ and $P_{2}=\left(x_{2}, y_{2}\right)$, the alignment $P_{1} \leftrightarrow P_{2}$ is satisfied iff $x_{1}=x_{2}$ and $y_{1}=y_{2}$.

Definition 6 (Line-Line Alignment) Given two lines $L_{1}=\left(X_{1}, Y_{1}\right)$ and $L_{2}=\left(X_{2}, Y_{2}\right)$, the alignment $L_{1} \leftrightarrow L_{2}$ is satisfied iff $X_{1}=X_{2}$ and $Y_{1}=Y_{2}$.

Definition 7 (Point-Line Alignment) Given a point $P=(x, y)$ and a line $L=(X, Y)$, the alignment $P \leftrightarrow L$ is satisfied iff $x X+y Y+1=0$.

As an aside, we note that there one other incidence-type relation that could be considered: a line can be oriented such that it is not incident to a given line - that is, it is parallel to a given line. While mathematically sound, in practice, this relationship can only be verified with infinite paper, and we will restrict our attentions to alignments that can be verified within a finite region of the paper.

Each of the HJAs can be viewed as a combination of one or more alignments. This leads naturally to the following definitions: 

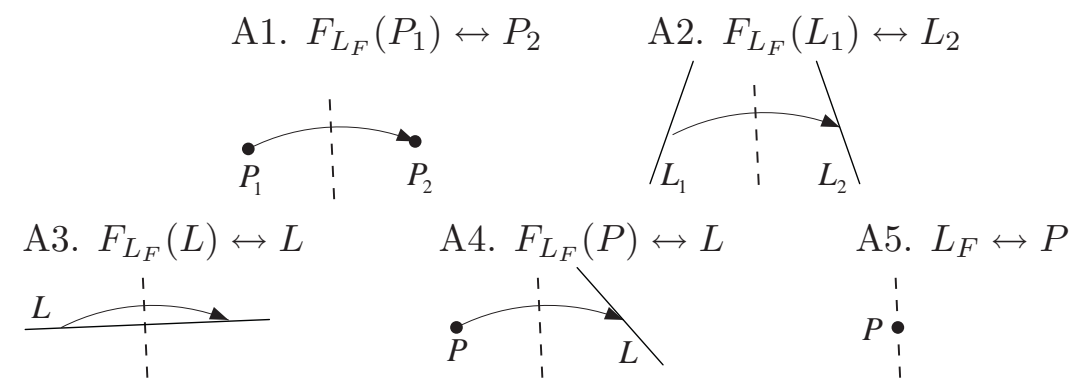

Figure 2: The five one-fold alignments. A1 and A2 define two equations each; A3-A5 define one equation each.

Definition 8 (One-Fold Axiom) $A$ one-fold axiom (1FA) is a minimal set of alignments that define a single fold line on a finite region of the Euclidean plane with a finite number of solutions.

We stipulate a minimal set to exclude redundant alignments that don't do anything. We stipulate a finite number of solutions because some combinations of alignments have multiple solutions. And we stipulate a finite region of the plane to exclude alignments that require infinite paper to verify.

With this definition, each of the HJAs can be seen to be a 1-fold origami axiom, and the question of completeness can now be precisely phrased: are there other 1FAs?

To set a condition on a fold line, one side of an alignment must be the folded image of a point or line. There are 5 possibilities with the 3 types of alignments for a single fold line $L_{F}$; each alignment defines 1 or 2 equations. These five one-fold alignments, denoted by A1-A5, are shown in Figure 2.

Note that A5 - aligning a point to the fold line - is equivalent to requiring that the folded image of a point be aligned to itself. That is, we could write $L_{F} \leftrightarrow P$ equivalently as $F_{L_{F}}(P) \leftrightarrow P$ (as was done in [21]).

The case of a line folded onto itself is distinct from a line folded onto another line in that only a single equation need be satisfied in the former.

Folding a point to a line $\left.\left(F_{L_{F}}(P) \leftrightarrow L\right)\right)$ is equivalent to folding a line to a point $\left.\left(F_{L_{F}}(L) \leftrightarrow P\right)\right)$ since they result in the same equations. We therefore consider the alignments $\left.F_{L_{F}}(P) \leftrightarrow L\right)$ and $F_{L_{F}}(L) \leftrightarrow P$ ) to be equivalent under folding.

Also, we do not consider the relationship of a line incident to the fold line since the goal is to define a fold line that does not already exist. 


\begin{tabular}{c|c|c|c} 
& $F_{L_{F}}\left(L_{1}\right) \leftrightarrow L_{1}$ & $F_{L_{F}}\left(P_{1}\right) \leftrightarrow L_{1}$ & $L_{F} \leftrightarrow P_{1}$ \\
\hline$F_{L_{F}}\left(L_{2}\right) \leftrightarrow L_{2}$ & $\mathrm{~N} / \mathrm{A}$ & O7 & O4 \\
$F_{L_{F}}\left(P_{2}\right) \leftrightarrow L_{2}$ & O7 & O6 & O5 \\
$L_{F} \leftrightarrow P_{2}$ & O4 & O5 & O1
\end{tabular}

Table 1: All possible pairs of single-equation alignments.

The fold line $L_{f} \equiv\left(X_{F}, Y_{F}\right)$ is defined by its two parameters and therefore has two degrees of freedom. Therefore, any combination of folds that specifies the fold line must consist of some combination of alignments that specify exactly two equations.

Each of the first two alignments in Figure 2 results in two equations that must be satisfied; thus, each in and of itself is sufficient to specify the fold line. And indeed, the alignment $F_{L_{F}}\left(P_{1}\right) \leftrightarrow P_{2}$ is equivalent to $\mathrm{O} 2$, while alignment $F_{L_{F}}\left(L_{1}\right) \leftrightarrow L_{2}$ is equivalent to O3.

The other 3 alignments only specify a single equation, which means that we require two such alignments to fully specify the two degrees of freedom of the fold line. We consider all possible pairs of the single-equation alignments in the following table. The points and lines (other than the fold lines) in each row and column are assumed to be distinct.

The top left combination (folding two different lines onto themselves) cannot be part of a valid axiom; if the two lines are nonparallel, then the equations are inconsistent, whereas if the lines are parallel, the equations are redundant and cannot be part of a minimal set. All other combinations correspond to existing HJAs - and we note that Justin/Hatori's 7th axiom is among them. Thus, Justin/Hatori's axiom can be defined in exactly the same way as the other 6 axioms. And, since we have considered all possible combinations of alignments, we have proved completeness; there are no more one-fold axioms to be found.

\section{Solving Equations with One-Fold Axioms using Lill's Method}

There is a classical method (Lill 1867, [28]) of using reflections to create the solutions to real polynomial equations. To solve the equation $x^{n}+a_{n-1} x^{n-1}+$ $\ldots+a_{0}$ by Lill's method, you form a right-angle path from the origin (point $O$ ) to a terminus (point $T$ ) in which the lengths and directions of the seg- 
(a)

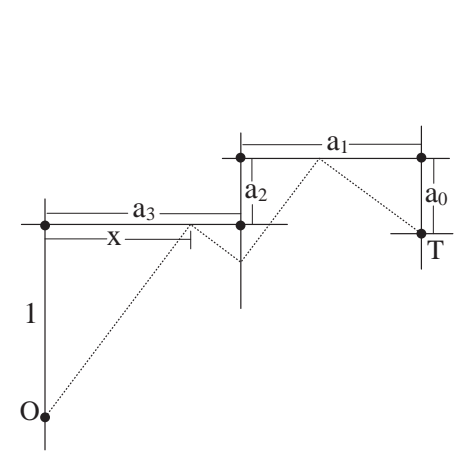

(b)

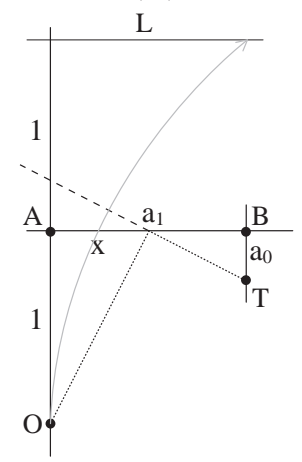

(c)

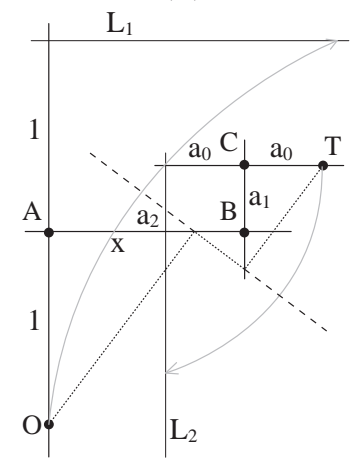

Figure 3: (a) Lill diagram for the quartic equation $x^{4}-a_{3} x^{3}+a_{2} x^{2}-a_{1} x-$ $a_{0}=0$. (b) Lill diagram and fold lines for solving the quadratic equation $x^{2}-a_{1} x-a_{0}=0$ using O5. (c) Lill diagram and fold lines for solving the cubic equation $x^{3}-a_{2} x^{2}+a_{1} x-a_{0}=0$ using O6.

ments are given by the coefficients of the equation, starting with the leading coefficient of 1 , ending with $a_{0}$, and at each junction, turning left or right depending on the sign of each coefficient (left=positive, right=negative). You then launch a line from $O$ at some angle that makes a right-angled turn at each successive line toward the next line, adjusting the initial slope until the final turn strikes point $T$. When that condition is satisfied, the first intersection point gives the desired solution. Lill's method works for arbitrarily high degree and lends itself nicely to equation solving via origami. Depending on the degree of the equation, we can use origami axioms to determine the correct bouncing strategy.

\subsection{Quadratics}

We are given three signed lengths determined from the polynomial: $O A, A B$, $B T$, each segment at right angles to the next, as in Figure 3(b). Construct an auxiliary line $L$ parallel to $A B$ on the opposite side to $A B$ from $O$ and of distance $O A$. Now use $\mathrm{O} 5$ to fold $O$ to $L$ so that the crease passes through $T$. 


\subsection{Cubics}

Using the diagram in Figure 3(c) made from arcs $O A, A B, B C, C T$, each at right angles, we construct auxiliary lines: $L_{1}$, parallel to $A B$ and opposite $O$ but of distance $O A ; L_{2}$, parallel to $B C$ and on the opposite side to $T$ but of distance $C T$. Using $\mathrm{O} 6$ we simultaneosly fold $O$ to $L_{1}$ and $T$ to $L_{2}$. This crease together with the its perpendiculars to $O$ and $T$ gives the desire bouncing strategy.

In the case of the cubic $x^{3}-a$ the solution using this method is quite similar to the method of [26]. We return to this method in $\S 5$ for solving higher degree equations with two or more folds.

\section{Two-Fold Axioms}

We now consider two-fold axioms: combinations of alignments that specify two simultaneous fold lines. We proceed in the same way as we did in the previous section. We consider the possible alignments between points, lines, fold lines, and their folded images. We then construct all possible combinations that specify two fold lines.

Definition 9 (Two-Fold Axiom) A two-fold origami axiom (2FA) is a minimal set of alignments that defines two simultaneous fold lines on a finite region of the Euclidean plane with a finite number of solutions.

There are a few complications when we consider two (or more) fold lines. First is a practical matter; physically creating a two-fold alignment requires that one smoothly vary the position of both folds until the various alignments are satisfied. With two simultaneous folds, any two nonparallel folds will eventually intersect and in the real world, intersecting folds bind at their intersection and cannot be smoothly varied in both position and angle. We will ignore this practical limitation for the moment.

Next, the number of possible alignments and combinations of alignments grows explosively with number of simultaneous folds (as we will see). In order to minimize the number of combinations to count, we will adopt several rules for equivalence and validity.

Definition 10 (Separability) A two-fold axiom is separable iff its alignments can be partitioned into two sets, each of which is a one-fold axiom. 
For example in [4] the trisection axiom afforded by Abe's method is given as a two-fold separable alignment combining $\mathrm{O} 2$ and O6: Given points $P$ and $Q$ and a line $l$ through $P$ then we can fold lines $L_{1}$ which reflects $P$ onto $Q$ and $L_{2}$ which reflect $Q$ onto $l$ and $P$ onto $L_{1}$.

Definition 11 (Equivalence Under Permutation) Two two-fold axioms are equivalent under permuation if their alignments are equivalent under permutation of their points, lines, and/or fold lines.

Definition 12 (Equivalence Under Folding) Two two-fold axioms are equivalent under folding if their alignments can be paired such that by application of $F_{L_{F 1}}$ or $F_{L_{F 2}}$ to both sides of one or more alignments makes them equivalent under permutation. Two axioms that are not equivalent under folding are distinct.

We will restrict our attention to enumerating non-separable distinct twofold axioms. To do this, we enumerate the distinct non-separable two-fold alignments, analogous to A1-A5 as defined in Figure 2. Figure 4 shows them all. For brevity, we name them AL1-AL10.

We include here only the alignments that lead to non-separable combinations. Any combination in which a single alignment fully specifies one of the fold lines will be separable.

Our ordering is chosen in roughly increasing degree of the underlying equations. If we denote the two fold lines by $F_{a}$ and $F_{b}$, the equations resulting from alignments AL1,AL8, and AL9 are symmetric under interchange of fold line while the equations resulting from alignments AL2, AL3, AL4, AL5, AL6, AL7, and AL10 are not. In forming combinations of the nonsymmetric alignments, we will append the letter $a$ or $b$ to distinguish the two forms; thus, for example. AL2a is the alignment shown in Figure 4; AL2b would act on the second fold line. We note that the potential alignment $F_{L_{a}}(L) \leftrightarrow F_{L_{b}}(L)$ results in the same equations as AL2a+AL2b, so we do not count it in our listing.

Alignment AL10 (which comes in both $a$ and $b$ varieties) is a bit unusual. All points appearing in other alignments are preexisting points, but the point $P_{L_{F_{a}}, L_{1}}$ appearing in AL10 is a "virtual point," the intersection of the first fold line $F_{L_{a}}$ with the existing line $L_{1}$. AL10 aligns this point with a second line $L_{2}$; if the two lines are the same $\left(L_{1}=L_{2}\right)$, then this alignment forces the intersection of the two fold lines with each other to lie on the given line. 


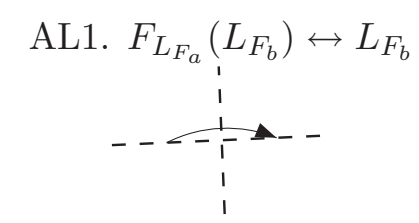

AL3. $L_{F_{a}} \leftrightarrow P$

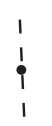

AL5. $F_{L_{F_{a}}}(P) \leftrightarrow L_{F_{b}}$

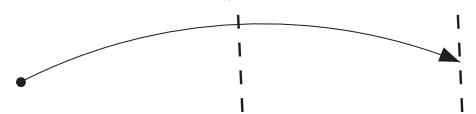

AL7. $F_{L_{F_{a}}}(P) \leftrightarrow F_{L_{F_{b}}}(L)$

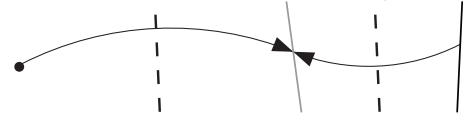

AL9. $F_{L_{F_{a}}}\left(L_{1}\right) \leftrightarrow F_{L_{F_{b}}}\left(L_{2}\right)$

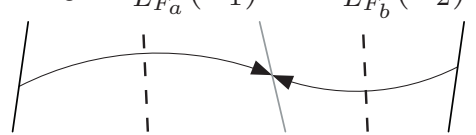

AL2. $F_{L_{F_{a}}}(L) \leftrightarrow L$

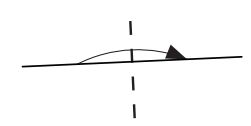

AL4. $F_{L_{F_{a}}}(L) \leftrightarrow L_{F_{b}}$

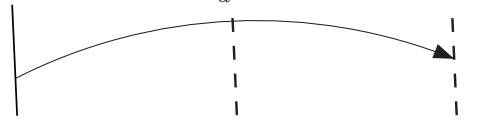

AL6. $F_{L_{F_{a}}}(P) \leftrightarrow L$

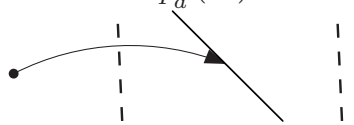

AL8. $F_{L_{F_{a}}}\left(P_{1}\right) \leftrightarrow F_{L_{F_{b}}}\left(P_{2}\right)$

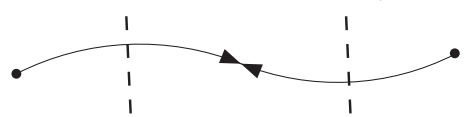

AL10. $F_{L_{F_{b}}}\left(P_{L_{F_{a}}, L_{1}}\right) \leftrightarrow L_{2}$

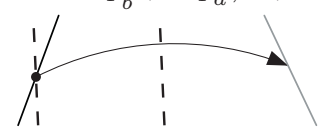

Figure 4: The 10 distinct two-fold alignments. 
Alignment $F_{L_{F_{b}}}\left(P_{L_{F_{a}}, L_{1}}\right) \leftrightarrow P_{1}$ is not listed because it can be decomposed into $F_{L_{F_{b}}}\left(L_{F_{a}}\right) \leftrightarrow P_{1}$ and $F_{L_{F_{b}}}\left(L_{1}\right) \leftrightarrow P_{1}$.

Each of these alignments leads to either one or two equations on the four degrees of freedom of the two fold lines, which implies that a valid two-fold axiom will consist of two, three, or four of these alignments. Including both $a$ and $b$ forms of the nonsymmetric alignments gives a total of 17 possible alignments for each element of a combination. That, in turn, gives $17 \times 17 \times 18 \times 18=93,636$ possible combinations to consider. However, those combinations include combinations that are over- and under-determined and combinations that are equivalent under permutation or folding. We constructed a computer-assisted enumeration using Mathematica, following this procedure:

1. Construct all possible combinations of 2,3 , or 4 of the two-fold alignments;

2. Eliminate duplicates equivalent under permutation or folding;

3. Construct symbolic equations for each remaining combination of alignments;

4. Eliminate combinations that did not lead to exactly 4 equations;

5. Construct the Jacobian for each set of 4 equations at a solution; eliminate combinations that did not have 4 singular values (indicating an inconsistent or under-determined set of equations).

We did this both with and without alignment AL10. Including AL10 gave 489 distinct combinations; leaving it out gave 203 combinations. Each combination is a distinct non-separable two-fold alignment, equivalent to the HJAs. To concisely identify a particular $2 \mathrm{FA}$, we adopt the following notation for a given combination of alignments. Begin with $A L$. Append the number of each alignment, including its $a / b$ suffix, in numerical order (but without repeating $\mathrm{AL}$ ). If an alignment appears more than once in combination, only the suffix is repeated. (This only happens with $a / b$ alignments.) Thus, for example, the 2FA denoted by AL6ab8 consists of alignments AL6a, AL6b, and AL8, and involves folding one point to a line using the first fold line; a point to a line using the second fold line; and bringing the image of a third and fourth points together using both fold lines. This $2 \mathrm{FA}$ and two others that also involve four points and two lines are shown in Figure 5. 
AL6ab8

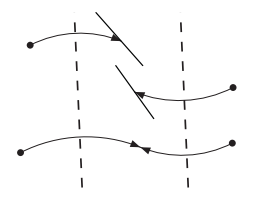

AL6a7a8

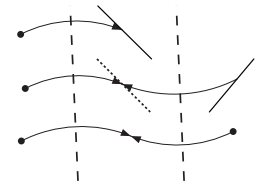

AL6a7b8

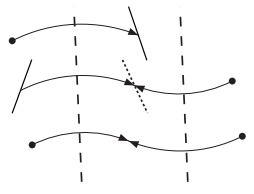

Figure 5: Three of the two-fold axioms.

Space does not permit a complete pictorial listing of the 2FAs here, but using our compact notation, we can provide a complete listing by symbol.

AL110aaa, AL110aab, AL12a10aa, AL12a10ab, AL12a10bb, AL12a3b10a, AL12a3b10b, AL12a3b7a, AL12a3b7b, AL12a6b10a, AL12a6b10b, AL12a6b7a, AL12a6b7b, AL12a7a10a, AL12a7a10b,

AL12a7aa, AL12a7ab, AL12a7b10a, AL12a7b10b, AL12a7bb, AL13a10aa, AL13a10ab, AL13a10bb, AL13ab10a, AL13ab7a, AL13a6b10a, AL13a6b10b, AL13a6b7a, AL13a6b7b, AL13a7a10a,

AL13a7a10b, AL13a7aa, AL13a7ab, AL13a7b10a, AL13a7b10b, AL13a7bb, AL16a10aa, AL16a10ab, AL16a10bb, AL16ab10a, AL16ab7a, AL16a7a10a, AL16a7a10b, AL16a7aa, AL16a7ab, AL16a7b10a, AL16a7b10b, AL16a7bb, AL17a10aa, AL17a10ab, AL17a10bb, AL17aa10a, AL17aa10b, AL17ab10a, AL810aa, AL810ab, AL910aa, AL910ab, AL10aaaa, AL10aaab, AL10aabb, AL2a810a, AL2a810b, AL2a910a, AL2a910b, AL2a10aaa, AL2a10aab, AL2a10abb, AL2a10bbb, AL2ab10aa, AL2ab10ab, AL2ab5a10a, AL2ab5a10b, AL2ab5ab, AL2ab5a7a, AL2ab5a7b, AL2ab7a10a, AL2ab7a10b, AL2ab7aa, AL2ab7ab, AL2a3b8, AL2a3b9, AL2a3b10aa, AL2a3b10ab, AL2a3b10bb, AL2a3b4a, AL2a3b4b, AL2a3b5a10a, AL2a3b5a10b, AL2a3b5aa, AL2a3b5ab, AL2a3b5a7a, AL2a3b5a7b, AL2a3b5b10a, AL2a3b5b10b, AL2a3b5bb, AL2a3b5b7a, AL2a3b5b7b, AL2a3b7a10a, AL2a3b7a10b, AL2a3b7aa, AL2a3b7ab, AL2a3b7b10a, AL2a3b7b10b, AL2a3b7bb, AL2a4a10a, AL2a4a10b, AL2a4a5b, AL2a4a6b, AL2a4a7a, AL2a4a7b, AL2a4b10a, AL2a4b10b, AL2a4b5a, AL2a4b6b,

AL2a4b7a, AL2a4b7b, AL2a5a8, AL2a5a9, AL2a5a10aa, AL2a5a10ab, AL2a5a10bb, AL2a5aa10a, A AL2a5ab6b, AL2a5ab7a, AL2a5ab7b, AL2a5a6b10a, AL2a5a6b10b, AL2a5a6b7a, AL2a5a6b7b, AL2a5b8, AL2a5b9, AL2a5b10aa, AL2a5b10ab, AL2a5b10bb, AL2a5bb10a, AL2a5bb10b, AL2a5bb6b, AL2a5bb7a, AL2a5bb7b, AL2a5b6b10a, AL2a5b6b10b, AL2a5b6b7a, AL2a5b6b7b, AL2a5b7a10a, AL2a5b7a10b, AL2a5b7aa, AL2a5b7ab, AL2a5b7b10a, AL2a5b7b10b, AL2a5b7bb, AL2a6b8, AL2a5b7a10b, AL2a5b7aa, AL2a5b7ab, AL2a5b7b10a, AL2a5b7b10b, AL2a5b7bb, AL2a6b8, AL2a6b7ab, AL2a6b7b10a, AL2a6b7b10b, AL2a6b7bb, AL2a7a10aa, AL2a7a10ab, AL2a7a10bb, AL2a7aa10a, AL2a7aa10b, AL2a7aaa, AL2a7aab, AL2a7ab10a, AL2a7ab10b, AL2a7abb, AL2a7b10aa, AL2a7b10ab, AL2a7b10bb, AL2a7bb10a, AL2a7bb10b, AL2a7bbb, AL3a810a, AL3a810b, AL3a910a, AL3a910b, AL3a10aaa, AL3a10aab, AL3a10abb, AL3a10bbb, AL3ab8, AL3ab9, AL3ab10aa, AL3ab10ab, AL3ab4a, AL3ab5a10a, AL3ab5a10b, AL3ab5aa, AL3ab5ab, AL3ab5a7a, AL3ab5a7b, AL3ab7a10a, AL3ab7a10b, AL3ab7aa, AL3ab7ab, AL3a4a10a, AL3a4a10b, AL3a4a5b, AL3a4a6b, AL3a4a7a, AL3a4a7b, AL3a4b10a, AL3a4b10b, AL3a4b5a, AL3a4b6b, AL3a4b7a, AL3a4b7b,

AL3a5a8, AL3a5a9, AL3a5a10aa, AL3a5a10ab, AL3a5a10bb, AL3a5aa10a, AL3a5aa10b, AL3a5aab, AL3a5aa6b, AL3a5aa7a, AL3a5aa7b, AL3a5ab10a, AL3a5ab10b, AL3a5abb, AL3a5ab6b, AL3a5ab7a, AL3a5a7aa, AL3a5a7ab, AL3a5a7b10a, AL3a5a7b10b, AL3a5a7bb, AL3a5b8, AL3a5b9, AL3a5b10aa, AL3a5b10ab, AL3a5b10bb, AL3a5bb10a, AL3a5bb10b, AL3a5bb6b, AL3a5bb7a, AL3a5bb7b, AL3a5b6b10a, AL3a5b6b10b, AL3a5b6b7a, AL3a5b6b7b, AL3a5b7a10a, AL3a5b7a10b, AL3a5b7aa, AL3a5b7ab, AL3a5b7b10a, AL3a5b7b10b, AL3a5b7bb, AL3a6b8, AL3a6b9, AL3a6b10aa, AL3a6b10ab, AL3a6b10bb, AL3a6b7a10a, AL3a6b7a10b, AL3a6b7aa, AL3a6b7ab, AL3a6b7b10a, AL3a6b7b10b, AL3a6b7bb, AL3a7a8, AL3a7a9, AL3a7a10aa, AL3a7a10ab, AL3a7a10bb,

AL3a7aa10a, AL3a7aa10b, AL3a7aaa, AL3a7aab, AL3a7ab10a, AL3a7ab10b, AL3a7abb, AL3a7b8, AL3a7b9, AL3a7b10aa, AL3a7b10ab, AL3a7b10bb, AL3a7bb10a, AL3a7bb10b, AL3a7bbb, AL4a8, AL4a9, AL4a10aa, AL4a10ab, AL4a10bb, AL4ab, AL4a5b10a, AL4a5b10b, AL4a5bb, AL4a5b6a, AL4a5b6b, AL4a5b7a, AL4a5b7b, AL4a6a10a, AL4a6a10b, AL4a6ab, AL4a6a7a, AL4a6a7b, AL4a6b10a, AL4a6b10b, AL4a6b7a, AL4a6b7b, AL4a7a10a, AL4a7a10b, AL4a7aa, AL4a7ab, AL4a7b10a, AL4a7b10b, AL4a7bb, AL5a810a, AL5a810b, AL5a910a, AL5a910b, AL5a10aaa, AL5a10aab, AL5a10abb, AL5a10bbb, AL5aa8, AL5aa9, AL5aa10aa, AL5aa10ab, AL5aa10bb, AL5aab10a, AL5aab10b, AL5aabb, AL5aab6a, AL5aab6b, AL5aab7a, AL5aab7b, AL5aa6a10a, AL5aa6a10b, AL5aa6ab, AL5aa6a7a, AL5aa6a7b, AL5aa6b10a, AL5aa6b10b, AL5aa6b7a,

AL5aa6b7b, AL5aa7a10a, AL5aa7a10b, AL5aa7aa, AL5aa7ab, AL5aa7b10a, AL5aa7b10b, AL5aa7bb, AL5ab8, AL5ab9, AL5ab10aa, AL5ab10ab, AL5ab6a10a, AL5ab6a10b, AL5ab6ab, AL5ab6a7a, AL5ab6a7b, AL5ab7a10a, AL5ab7a10b, AL5ab7aa, AL5ab7ab, AL5a6a8, AL5a6a9, AL5a6a10aa, AL5a6a10ab, AL5a6a10bb, AL5a6ab10a, AL5a6ab10b, AL5a6ab7a, AL5a6ab7b, AL5a6a7a10a, AL5a6a7a10b, AL5a6a7aa, AL5a6a7ab, AL5a6a7b10a, AL5a6a7b10b, AL5a6a7bb, AL5a6b8, AL5a6b9, AL5a6b10aa, AL5a6b10ab, AL5a6b10bb, AL5a6b7a10a, AL5a6b7a10b, AL5a6b7aa, AL5a6b7ab, AL5a6b7b10a, AL5a6b7b10b, AL5a6b7bb, AL5a7a8, AL5a7a9, AL5a7a10aa, AL5a6b7ab, AL5a6b7b10a, AL5a6b7b10b, AL5a6b7bb, AL5a7a8, AL5a7a9, AL5a7a10aa, AL5a7ab10b, AL5a7abb, AL5a7b8, AL5a7b9, AL5a7b10aa, AL5a7b10ab, AL5a7b10bb, AL5a7bb10a, 
AL5a7bb10b, AL5a7bbb, AL6a810a, AL6a810b, AL6a910a, AL6a910b, AL6a10aaa, AL6a10aab, AL6a10abb, AL6a10bbb, AL6ab8, AL6ab9, AL6ab10aa, AL6ab10ab, AL6ab7a10a, AL6ab7a10b, AL6ab7aa, AL6ab7ab, AL6a7a8, AL6a7a9, AL6a7a10aa, AL6a7a10ab, AL6a7a10bb, AL6a7aa10a, AL6a7aa10b, AL6a7aaa, AL6a7aab, AL6a7ab10a, AL6a7ab10b, AL6a7abb, AL6a7b8, AL6a7b9,

AL6a7b10aa, AL6a7b10ab, AL6a7b10bb, AL6a7bb10a, AL6a7bb10b, AL6a7bbb, AL7a810a,

AL7a810b, AL7a910a, AL7a910b, AL7a10aa, AL7a10aab, AL7a10abb, AL7a10bbb, AL7aa10aa, AL7aa10ab, AL7aa10bb, AL7aaa10a, AL7aaa10b, AL7aab10a, AL7aab10b, AL7ab10aa, AL7ab10ab

This leads naturally to the question: what good are these? The most complex of the HJAs, axiom O6, imposes a cubic equation on the parameterization of the fold line; this condition enables the solution of the general cubic equation and various related problems. Folding a 2FA imposes higherorder equations on the parameterizations of the fold lines; thus, a 2FA can potentially be used to solve higher-order polynomial equations.

We note that the previously-mentioned angle quintisection [23] requires a two-fold alignment at one step. Specifically, the required 2FA is AL3a5b6b7b. Performing an angle quintisection requires the solution of a particular irreducible quintic equation; thus, this 2FA (and others) allows the solution of at least some quintic polynomials by origami. The question of which $2 \mathrm{FAs}$ allow this solution, and which still higher-order equations could be addressed by 2 FAs, is the topic of the next section.

\section{Solving Equations with Two-Fold Axioms}

In order to fix a line it needs to be specified by two conditions; so two folds need four conditions to determine the alignment. Each two-fold alignment yields a system of four equations in four variables with each equation of degree at most 4.

Of the first 310 2FAs, 303 of them contain at least one equation of degree 1 in two variables (ending with AL3a7bbb); by eliminating a variable we can reduce the system to three equations of degree at most 4 in three variables. Of these, 85 systems involve two equations of degree 1 so by further elimination we are led to two equations in two unknowns; the solutions to each of these equations is a curve in the plane and the simultaneous solutions give the coordinates for the two fold lines. By Bezout's theorem the product of the two degrees is the number of (complex) solutions counted with multiplicity.

Considering all systems, the number of solutions is much less than the theoretical upper bound of 192 (the product of degrees); we obtain at most 21 solutions in the worst case using Groëbner basis calculations (see e.g.[30]) in the program Magma. 
In our equations the first fold is $(X, Y)$ and the second fold is $(Z, W)$. The coefficients of our equations belong to the ring $A$ of rational functions in variables determined by the generic points and lines given by the data of the alignment conditions. The fold conditions give an ideal

$$
<a(X, Y, Z, W), b(X, Y, Z, W), c(X, Y, Z, W), d(X, Y, Z, W)>
$$

in $A[X, Y, Z, W]$ which describes an affine variety of dimension 0 (as a consequence of the non-singularity of the Jacobian of $a, b, c, d)$. We apply Groëbner basis methods (after specializing the point and line coordinates in $A$ to rational values). The Groëbner basis method gives another set of ideal generators of the following form

$$
<f_{0}(W), X-f_{1}(W), Y-f_{2}(W), Z-f_{3}(W)>.
$$

The degree of $f_{0}$ is called the complexity, denoted $c x$. The degrees of the other polynomials generators in this ideal are smaller than $c x$. The complexity is our measure of the number of different crease patterns which fulfill the given alignment conditions. For different choices of given points and lines in the two-fold alignment we get different polynomials; for AL3ab9 the polynomial $f_{0}$ is of degree 4 , but not all polynomials of degree 4 can arise in this way.

\section{$6 \quad$ Geometry Examples}

We explore some of the geometry involved in selected two-fold alignments and their complexity.

\subsection{Trisection}

AL4ab $c x=3$ : For non-parallel given lines, this alignment gives the trisections of the angle between the given lines as one of the creases and the other crease is the bisector of the first crease with the remote line. For parallel given lines, the alignment gives the folds for the trisection of the segment perpendicular to the given lines. 


\subsection{Conics}

\subsubsection{Two Circles: AL3ab*_-17 two-folds}

AL3ab9, $c x=4$ : The fold line and point for AL3a or AL3b can be viewed as a diameter and center of a circle. The folds reflect given lines to other tangents of the circles. Thus, this folds the (at most) 4 common tangents to two circles.

The other alignments of this type have complexity at most 8; the basis polynomial $f_{0}$ for AL3ab7ab factors as a quadratic and sextic; others of this type have small complexity.

\subsubsection{Circle and Parabola: AL3a6b-29 two-folds}

AL3a4a6b $c x=4$ : The second crease can be viewed as a tangent to a parabola with the point and line of AL6b as its focus and directrix; the first crease is a diameter to a circle. The given line is folded to a tangent to a circle and is a tangent of the parabola; thus alignment creates the (at most) 4 tangents to a circle and parabola. A method for solving cetain quartic polynomials by folding the common tangents to a circle and parabola has been discussed in [6]. The method discussed there however does not use a two-fold.

The alignments of this type have complexity at most 12 . The highest degree factor of $f_{0}$ by the Groëbner basis calculations yield: AL3a6b4b of degree 3; AL3a6b5aa of degree 4; AL3a6b5ab of degree 6; AL3a6b5a7a of degree 6 ;AL3a6b5a7b of degree 8;AL3a6b5bb of degree 3; AL3a6b5b7a of degree 8; AL3a6b5b7b of degree 7; AL3a6b8 of degree 4; AL3a6b9 of degree 6; AL3a6b7aa of degree 10; AL3a6b7ab of degree 10; AL3a6b7bb of degree 8; AL3a6b5a10a of degree 8; AL3a6b7a10b of degree 10.

\subsubsection{Two Parabolas: AL6ab* and Solving Quartics}

The nine alignments involving AL6a and AL6b have a complexity at most 18 . However, the highest degree factor of $f_{0}$ by the Groëbner basis calculations yield: AL6ab4a of degree 5; AL6ab5aa of degree 5; AL6ab5ab of degree 10; AL6ab5a7a of degree 10; AL6ab5a7b of degree 12; AL6ab9 of degree 8; AL6ab7aa of degree 11; AL6ab7ab of degree 15; AL6ab7a10a of degree 14.

The alignment AL6ab9 has the highest factor of the polynomial $f_{0}$ with Galois group of order 1152 in general. By using a repeated line we reduce 

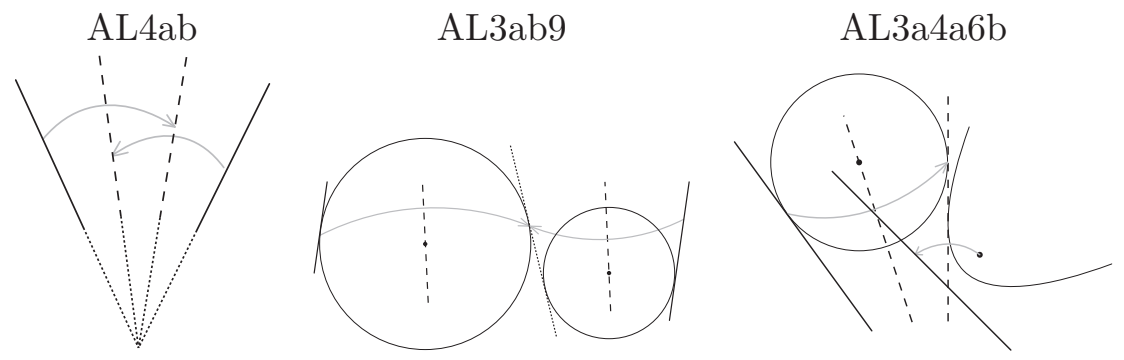

Figure 6: Three 2FAs. AL3ab9 and AL3a4a6b are shown with their associated conic sections.

the complexity to 4 and can then solve the general quartic by using Lill's method. We can create this construction using alignment AL6ab9 with the two parabolas and the (repeated) line $B C$ as shown in Figure 7 . Using $O A$, $A B, B C, C D, D T$ we consider two parallel parabolas with vertices at $A$ and $D$ and foci at $O$ and $T$ respectively. The two directrices of the parabolas are easily constructed atop the Lill path. We then fold points $O$ and $T$ onto their respective directrices so that the two fold lines intersect at a point on line $B C$. The two creases give two reflections which is a rotation; since we use the same line in the alignment the creases must be perpendicular and meet on $B C$. Thus $O X, X Y, Y Z, Z T$ is the sequence of right angled bounces with $X, Z$ on the lines $A B, C D$ respectively and on the respective creases. The distance from point $A$ to point $X$ is the desired solution.

Notice that if two folds are perpendicular and meet at $P$ and a line $L$ passes through $P$ then the reflection of $L$ in the first and reflection of $L$ in the second are the same line; conversely if $L$ reflects in two folds to the same line then the folds are perpendicular and meet at a point on the line $L$.

We note in passing that the two fold lines meet at right angles (which could be enforced by AL1) and the intersection of the fold lines lies on line $B C$ (which could be enforced by AL10); this particular construction could be equivalently created by AL16ab10a. 


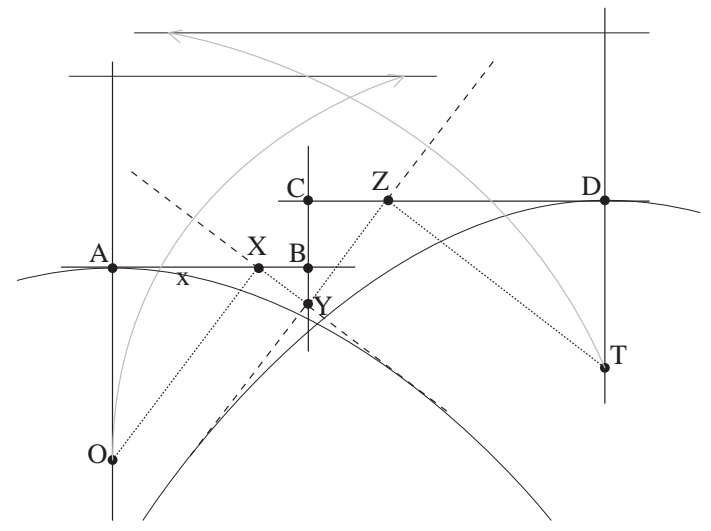

Figure 7: Solving a quartic equation by origami and Lill's method. The two associated parabolas are also shown.

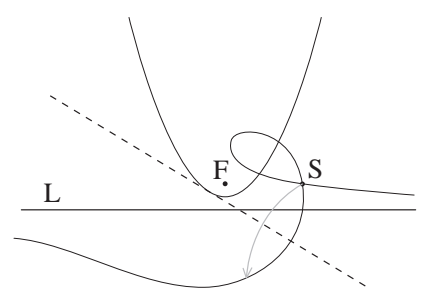

Figure 8: The image of a point $S$ across all fold lines that are tangents to a parabola defined by focus $F$ and directrix $L$ traces out an origami cubic curve.

\section{Higher Origami: Examples of degrees 5-8}

\subsection{Origami Cubic Curve}

Reflect a point $S$ in the tangents to a parabola; the locus created is the origami cubic curve. It is singular at $S$ and is circular, i.e., the cubic part of its equation is $\left(x^{2}+y^{2}\right)(a x+b y)$ when the directrix of the parabola has equation $a x+b y=c$. An example is shown in Figure 8.

In the 1-fold axiom $\mathrm{O} 7$ we fold a common tangent to two parabolas. Call the foci $F$ and $S$ and the directrix of the first is $L$, the directrix of the second is $M$. We can think of this as reflecting $S$ across the tangents of the parabola so as to land on $M$. In other words we are intersecting $M$ with the origami cubic curve and getting three intersections [3]. 


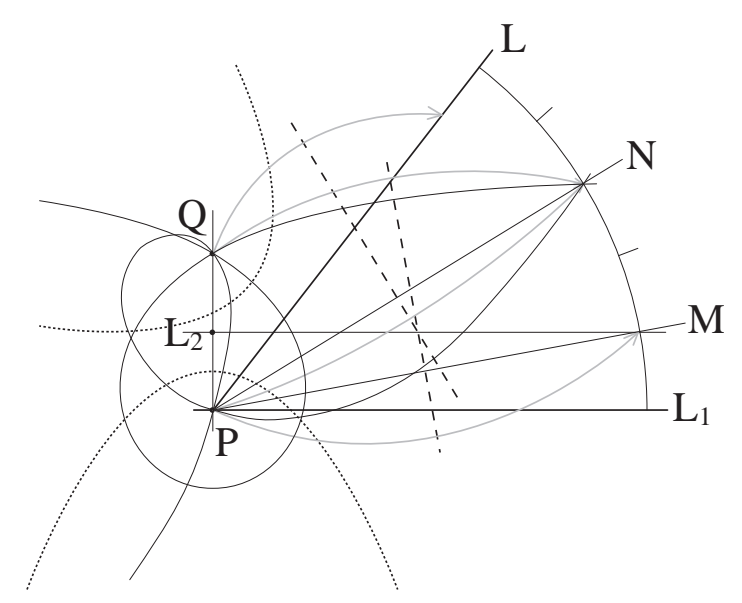

Figure 9: Quintisection by AL6ab8

\subsubsection{Septics and Quintics-AL6ab8}

The two-fold alignment AL6ab8 means that we are given two parabolas by foci and directrix $F_{a}, L_{a}, F_{b}, L_{b}$ and two other points $S_{a}, S_{b}$ which reflect across the parabola tangents so as to be coincident. That is the same as locating the intersection of two origami cubics $J_{a}$ determined by $F_{a}, L_{a}, S_{a}$ and $J_{b}$ determined by $\left.F_{b}, L_{b}, S_{b}\right)$. Two cubics will meet in general in 9 points but here both cubics are circular so they already meet at the 2 circular points. Therefore there are generally seven other intersections; that is the complexity is 7 .

If we enforce other coincidences, like the singular point $S_{a}$ also lies on $J_{b}$, then there will be a common singular point counting for a double intersection, so the complexity is reduced to 5 .

In this way we can, by taking the intersection of the two cubic curves, obtain a quintic $f_{0}$ which can be solved by means of AL6ab8. It is not known whether all field extension of the rational numbers of degree 5 can be generated by a root of such an $f_{0}$; however by various choices of points and lines we can arrange for both solvable and non-solvable Galois groups. One of the solvable cases gives a second method for quintisection.

\subsubsection{Quintisection}

Here we are given lines $L_{1}$ and $L$. We want to fold an intermediate angle determined by $N$ and its trisection by $M$ so that the reflection of $M$ across 
$N$ lands on $L$. This is the same as the use of Maclaurin's trisectrix (Abe's origami trisection) and a simultaneous Archimedes trisection (by neusis). We do both to get a quintisection. It amounts to an intersection of two origami cubics as described above. In Figure 7.1.2 the angle between $L$ and $L_{1}$ is quintisected using the intersection of two cubics. The cubic with singularity at $Q$ is the trisectrix. The cubic with singularity at $P$ is a focal cubic. The angle between $N$ and $L_{1}$ is trisected by $M$ using properties of the trisectrix; $N$ is the bisector of $L$ and $M$ using properties of focal cubics.

\subsection{Origami Cubic Line Curve}

An analogue of the origami cubic curve is obtained by reflecting a line in the tangents to a parabola and then dualizing this line in a parabola or other relevant conic; this yields a singular cubic curve which we call an origami line cubic curve.

\subsubsection{AL4a6ab, $c x=5$}

Here we want the reflection of given line $L_{2}$ using the focus directrix $P_{1}-L_{1}$ of AL6a to land on the fold made by $Q_{1}-M_{1}$ using AL6b. Let $\mathcal{K}$ be the parabola made by the focus-directrix $Q_{1}-M_{1}$. We dualize the origami line cubic made by $P_{1}, L_{1}, L_{2}$ in $\mathcal{K}$. The intersections of this singular cubic with $\mathcal{K}$ are precisely those places where reflections of $L_{2}$ give tangents to $\mathcal{K}$. Thus the 6 possible intersections of the conic $\mathcal{K}$ and the origami line curve give the possible two-folds. Since the point at infinity on the conic is one of the common points the solutions are given by a quintic.

\subsubsection{AL6ab9, $c x=8$}

In this construction we create two line curves by reflections of lines $L_{2}$ and $M_{2}$ in the tangents of $P_{1}-L_{1}$ and $Q_{1}-M_{1}$. We are looking for the coincidences of the two line curves. If we dualize these in the same parabola $\mathcal{K}$ then the two cubics will meet in at most 9 points; thus, there are at most 9 possible configurations for a given set of data of lines and points. However there is a common point at infinity (the parabolas) for these two cubics so there are only 8 distinct points of intersection and thus 8 possible two-fold configurations. 


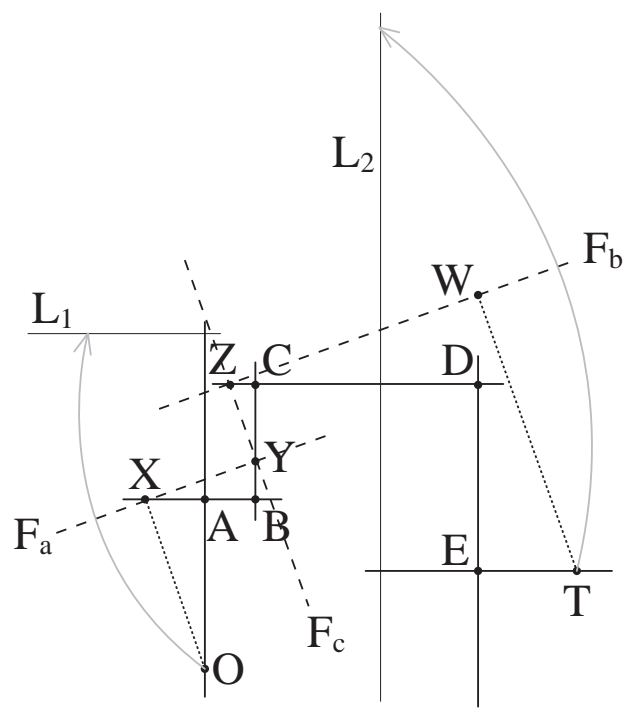

Figure 10: Solving the Quintic with 3-Fold

\section{Three-Folds and More}

\section{Solving the General Quintic By Lill's Method}

While selected quintics can be solved by 2FAs, we have not yet found a solution to the general quintic. However, the general solution is possible using three simultaneous folds. Using sides $O A, A B, B C, C D, D E, E T$ at right angles we create the Lill solution $O X, X Y, Y Z, Z W, W T$ at right angles with $X, Y, Z, W$ on the sides of the diagram (Figure 8).

Fold $O$ to $L_{1}$ on $F_{a}$ where $L_{1}$ is parallel to $A B$ passing thru the reflection of $O$ in $A B$; this is AL6a. This fold line meets $A B$ at $X$ and $B C$ at $Y$. Fold $T$ to $L_{2}$ on $F_{b}$ where $L_{2}$ is parallel to $D E$ passing through the reflection of $T$ in $D E$; this is AL6b. Intersection with $D E$ is $W$ and intersection with $C D$ is $Z$.

At the same time form a third fold $F_{c}$ that is perpendicular to $F_{a}$ (AL1) so that the fold intersection lies on $C D$ (AL10) and is perpendicular to $F_{b}$ (AL1) so that the fold intersection lies on $B C$ (AL10). Lines $O X$ and $W T$, plus segments of the three fold lines, complete the Lill path and give a solution to the general quintic. 


\section{More Folds, Higher Degree Equations}

Before proceeding, we note that the concept can be more broadly generalized:

Definition 13 ( $N$-Fold Axiom) An $N$-fold axiom is a minimal set of alignments between sets of points, lines, and their folded images that defines a finite number of sets of $N$ fold lines on a finite region of the Euclidean plane.

More generally, this technique can be used to solve an arbitrary polynomial equation using Lill's method. An $n$ th-degree equation has a Lill diagram for which the solution path consists of $n$ segments with $n-1$ turns. As in the preceding example, we use AL6 for the first and last segments of the path and $n-2$ folds for the intermediate segments, applying alignments AL1 and AL10 to enforce right-angle turns and turns lying on specified lines, respectively, for the $n-3$ intermediate turns. The number of equations to be satisfied are $2 \times(n-3)+2=2(n-2)$, matching the available DOF of $n-2$-folds. Thus:

Theorem 1 Every polynomial equation of degree $n$ with real solutions can be solved by $n-2$ simultaneous folds.

The proof follows from the argument in the preceding paragraph. It would be interesting to know if equations of degree $n$ which can be solved by radicals can be solved in general using less than $n-2$-folds. This is certainly true for $n=4$ and unknown for $n=5$.

\section{References}

[1] Hisashi Abe, described in British Origami, no. 108, p. 9, 1984

[2] R. C. Alperin, "A Mathematical Theory of Origami Constructions and Numbers," New York J. Math., 6, 119-133, 2000; available at http://nyjm.albany.edu

[3] R. C. Alperin, "A Grand Tour of Pedals of Conics," Forum Geometricorum, 4, 143-151, 2004.

[4] R. C. Alperin, "Trisections and Totally Real Origami," American Math. Monthly, vol. 112, no. 3, 200-211, March 2005. 
[5] David Auckly and John Cleveland, "Totally Real Origami and Impossible Paper Folding," American Math. Monthly, vol. 102, no. 3, pp. 215-226, March 1995.

[6] B. Carter Edwards and Jerry Shurman, "Folding Quartic Roots," Mathematics Magazine, 74(1), 19-25 (2004)

[7] Koji Fusimi, "Trisection of angle by Abe," Saiensu supplement, October, 1980, p. 8 .

[8] C. F. Gauss, Disquisitiones Arithmeticae, Leipzig, 1801, translated from the Latin by A. A. Clarke, Yale University Press, New Haven, CT , 1965.

[9] Robert Geretschläger, "Euclidean Constructions and the Geometry of Origami," Mathematics Magazine, vol. 68, no. 5, December, 1995, pp. $357-71$.

[10] Robert Geretschläger, "Folding the Regular Triskaidekagon," presented at AMS Joint Mathematics Meeting, Baltimore, MD., January 9, 1998.

[11] Robert Geretschläger, "Folding the Regular 19-gon," presented at AMS Joint Mathematics Meeting, Baltimore, MD., January 9, 1998.

[12] Robert Geretschläger, "Solving Quartic Equations in Origami," presented at AMS Joint Mathematics Meeting, Baltimore, MD., January 9, 1998.

[13] Koshiro Hatori, http://www.jade.dti.ne.jp/ ^hatori/library/conste.html, 2002.

[14] Thomas Hull, "A Note on Impossible Paperfolding," The American Mathematical Monthly, vol. 103, no. 3, March, 1996.

[15] Humiaki Huzita, "Axiomatic Development of Origami Geometry," Proceedings of the First International Meeting of Origami Science and Technology, Humiaki Huzita, ed., 1989, pp 143-158.

[16] Humiaki Huzita, "A possible example of system expansion in origami geometry," Proceedings of the First International Meeting of Origami Science and Technology, Humiaki Huzita, ed., 1989, pp 53-70. 
[17] Humiaki Huzita, "A problem on the Kawasaki theorem," Proceedings of the First International Meeting of Origami Science and Technology, Humiaki Huzita, ed., 1989, pp. 159-164.

[18] Humiaki Huzita, "The trisection of a given angle solved by the geometry of origami," Proceedings of the First International Meeting of Origami Science and Technology, Humiaki Huzita, ed., 1989, pp. 195-214.

[19] Humiaki Huzita and Benedetto Scimemi, "The Algebra of PaperFolding (Origami)," Proceedings of the First International Meeting of Origami Science and Technology, Humiaki Huzita, ed., 1989, pp. 215222.

[20] Humiaki Huzita, "Understanding Geometry through Origami Axioms," Proceedings of the First International Conference on Origami in Education and Therapy (COET91), J. Smith ed., British Origami Society, 1992, pp. 37-70.

[21] Jacques Justin, "Resolution par le pliage de l'equation du troisieme degre et applications geometriques," Proceedings of the First International Meeting of Origami Science and Technology, Humiaki Huzita, ed., 1989, pp. 251-261.

[22] Jacques Justin, described in British Origami, no. 107, pp. 14-15, 1984.

[23] Robert J. Lang, http://www.langorigami.com/science/quintisection

[24] Robert J. Lang, "Origami Approximate Geometric Constructions,", in Tribute to a Mathemagician, Barry Cipra, Erik D. Demaine, Martin L. Demaine, and Tom Rodgers, eds., A K Peters, 2004.

[25] George E. Martin, Geometric Constructions, Springer-Verlag, 1998, pp. $145-159$.

[26] Peter Messer, "Problem 1054," Crux Mathematicorum, vol. 12, no. 10, December, 1986. http://web.merrimack.edu/hullt/geoconst.html, 2003 .

[27] F. Morley and F. V. Morley, Inversive Geometry, Chelsea Publishing, New York, 1954. 
[28] M. Riaz, "Geometric Solutions of Algebraic Equations," American Math. Monthly, vol. 69, no. 7, 654-658, September, 1962.

[29] B. Scimemi, "Draw of a regular heptagon by the folding," Proceedings of the First International Meeting of Origami Science and Technology, Humiaki Huzita, ed., 1989, pp 71-78.

[30] B. Sturmfels, Solving Systems of Polynomial Equations, American Math. Society, CBMS vol. 97, 2002. 